\title{
EXPECTATIVAS QUE POSEEN LOS DOCENTES UNIVERSITARIOS DE CARRERAS DE PEDAGOGÍA EN RELACIÓN AL USO DE LAS TIC
}

\section{EXPECTATIONS OF TEACHING DEGREE UNIVERSITY PROFESSORS IN THE USE OF ICT}

\author{
Dr. Marcelo Humberto Rioseco Pais ${ }^{1}$ \\ mrioseco@ucm.cl \\ Rosabel Roig Vila² \\ Rosabel.roig@ua.es
${ }^{(1)}$ Universidad Católica del Maule. Facultad de Ciencias de la Educación Avd. San Miguel 3605, Talca (Chile).
${ }^{(2)}$ Universidad de Alicante. Facultad de Educación. Dpto. de Didáctica General y Didácticas Específicas Ap. Correos 99, 03080, Alicante (España).

Este artículo presenta un estudio cualitativo realizado en la Facultad de Educación de la Universidad Católica del Maule (Chile), en relación a las expectativas de los académicos en torno a la integración de las TIC en educación. Se expone el diseño metodológico basado en el análisis de contenido y la codificación abierta de la teoría fundamentada, y en el cual se ha utilizado el programa de análisis cualitativo ATLAS.ti (CQDAS). En general, los académicos poseen altas expectativas respecto a los efectos que se producen al utilizar las TIC respecto a diversos aspectos.

Palabras claves: Tecnología educacional, recursos educacionales, investigación sobre la educación, innovación educacional, eficacia de la educación.

This article presents a qualitative study carried out in the School of Education at the Catholic University of Maule (Chile) in relation to scholars' expectations on the integration of ICT in education. Methodological design, based on content analysis and open coding of grounded theory, is exposed; and which uses the qualitative analysis software ATLAS.ti (CQDAS). In general, the scholars have high expectations on the effects to be produced when using ICT in different aspects.

Key words: Educational technology, educational resources, educational research, educational innovation, effectiveness of education. 


\section{Introducción.}

Durante las últimas décadas, las Tecnologías de la Información y de la Comunicación (TIC) se han desarrollado y expandido de forma exponencial. No sólo las economías y los países desarrollados han visto cómo se transforman sus procesos productivos, de generación de conocimiento y de transmisión cultural a partir de un sinfín de recursos digitales presentes en la vida cotidiana, sino que se trata, más bien, de un fenómeno planetario. Muchos de estos cambios se han producido a nivel de objetos y de entornos materiales tangibles. Sin embargo, muchos otros tienen que ver con transformaciones subjetivas, maneras de percibir y de estructurar el mundo que nos rodea. Es en este ámbito donde entran en juego las expectativas que son, en última instancia, las que dan sentido y dirección a todo el proceso de transformación social y humana, incluyendo, por supuesto, al espacio objetivo que se modifica junto a las personas.

\section{Fundamentación del problema de estu- dio.}

Hoy en día se dispone de una cantidad importante de literatura que explica desde diversas perspectivas el modo y la dimensión en que son utilizadas las TIC en los ámbitos educativos. Así, Olvera-Lobo y Benítez-deVendrell (2008) desarrollaron un estudio titulado «Aproximación a las actitudes y percepciones de los usuarios ante las tecnologías de la información». Para su investigación utilizaron el modelo de Aceptación de la Tecnología (TAM o Technology Acceptance Model), un modelo de explicación de actitudes frente a la tecnología posterior al TRA (Theory of Reasoned Action), y a la escala de actitud desarrollada por Loyd y Gressard (1984). En su análisis concluyeron que una puesta en práctica eficaz de la tecnología depende, principalmente, de una actitud positiva de los usuarios hacia ella, sin importar demasiado lo sofisticada que pueda ser esta tecnología.

En una investigación más reciente y enfocada en las actitudes de los profesores universitarios, Tejedor, García-Valcárcel y Prada (2009) desarrollaron un estudio titulado « Medida de actitudes del profesorado universitario hacia la integración de las TIC» según el cual es posible identificar estas actitudes entre dos polos extremos: tecnofobia, es decir, el rechazo del uso de las máquinas, y tecnofilia, como la emoción a sentirse incorporado al mundo de la tecnología. Las principales causas de las actitudes negativas se relacionan con factores tales como: resistencia al cambio, deficiencias de formación en el uso de las tecnologías, autoestima y el grado de frustración, y la visión del ordenador como sustituto del profesor.

Por otra parte, muchos de los factores que beneficiarían una actitud positiva de los docentes tienen que ver con una incidencia de las TIC en el rendimiento de los alumnos (Ifeoma, 2014), o con una percepción positiva por parte de todo el alumnado, independientemente de su edad, con respecto a las TIC (Yau \& Cheng, 2012). De esta forma, es posible afirmar que la integración de las TIC en la práctica del aula requiere profundos cambios en los sistemas educativos, tanto en las creencias y actitudes del profesorado, como en las políticas educativas y sus contextos, así como en las innovaciones que se lleven a cabo (Oostveen, Muirhead \& Goodman,2011; Saeed, Yang \& Sinnappan, 2009).

Las actitudes de los docentes frente a la 
integración curricular de las TIC, entendiendo la actitud como una «reacción adquirida, más o menos emocional, hacia un estímulo cualquiera» (Pieron, 1993), se basa en las creencias y las expectativas que un sujeto, un grupo de sujetos o, incluso, una sociedad posee frente a este tipo de herramientas como recursos para apoyar el proceso de enseñanza-aprendizaje. Ahora bien, ¿en qué condición se está actualmente en lo que se refiere a esta integración curricular de las TIC, después de que muchos gobiernos hayan adoptado la adquisición y uso de las TIC en las escuelas como políticas públicas en el ámbito educativo? ¿Cuántas de estas expectativas se han cumplido realmente y cuántas no?

En este contexto, Benavides y Francesc (2007) han elaborado, auspiciados por la OCDE, una visión panorámica de las relaciones entre TIC y la educación escolar, atendiendo, principalmente, a su vinculación con la innovación educativa y sus implicaciones políticas, institucionales y pedagógicas en los países iberoamericanos. Los autores establecen un marco de expectativas en torno a los aportes pedagógicos de las TIC, mencionando cuatro ejes fundamentales: a) desarrollo económico: importancia de contar con recursos humanos capacitados en TIC, capaces de adaptarse en una sociedad en red; b) equidad y justicia social: la reducción de la brecha digital como un factor que ayuda a generar igualdad de oportunidades; c) cambio pedagógico: las TIC como elemento que promueve el cambio pedagógico en la sociedad del conocimiento; d) calidad en el aprendizaje: las TIC como un recurso para mejorar la calidad de los aprendizajes.

Según estos autores, han pasado casi tres décadas desde que se creó el primer ordenador personal y más de una década en que Internet se convirtió en un recurso de acceso público yes una opinión general que los progresos no han tenido ni la rapidez ni los impactos que se esperaba, tanto de forma general, como en colectivos específicos (Chiner \& Cardona, 2012, 2013; López-Torrijo \& Mengual-Andrés, 2014). Por una parte, el acceso a las tecnologías digitales ha tenido un crecimiento espectacularmente rápido. Por otra parte, el nivel de uso de las TIC en los centros escolares es bajo y la frecuencia dista mucho de ser diaria.

Cuando se describe el impacto, la importancia y el desarrollo de los recursos TIC en educación prevalece un punto de vista centrado en aspectos externos al sujeto, tales como la cobertura y masificación que poseen determinados aparatos y programas de software. Por el contrario, cuando la incorporación de las TIC no genera los resultados que se esperan en términos académicos y de aprendizajes, las dificultades suelen identificarse con la falta de motivación, de habilidades y de conocimientos técnicos por parte de profesores y estudiantes (Cabero \& Marín, 2012; Kapoor \& Sharma, 2013; Zelick, 2013). Pareciera, sin embargo, que el problema va más allá de todo esto. Es probable que se requiera de una reflexión más profunda en torno a las significaciones construidas socialmente en relación al uso de la tecnología desde cualquier perspectiva, incluso desde los primeros años de escolarización (Marín \& Martín-Párraga, 2014).

En este sentido, se hace necesario reflexionar acerca de las creencias que operan y que ayudan a reproducir un modelo racionalistarepresentacionista, fuertemente incorporado en los procesos de educación formal y que incide en la manera en que son utilizados los recursos TIC en los diferentes niveles del sistema educativo.

Las actitudes hacia las TIC que, en térmi- 
nos generales, pueden ser positivas o negativas, están asociadas a disposiciones específicas que expresan profesores y estudiantes cuando se trata de utilizar estos recursos en el proceso de enseñanza-aprendizaje (Almekhlafi \& Almeqdadi, 2010; Hayes \& Orellana, 2011; Koc \& Bakir, 2010). El estudio que se presenta a continuación tiene, pues, como objetivo principal, aproximarse, desde un enfoque cualitativo, a la manera en que los académicos a cargo de la formación inicial docente dan significado a la integración de las TIC en el currículum, a través de las expectativas que tienen hacia ellas, como recursos de apoyo a la formación.

\section{Diseño metodológico.}

Esta investigación fue desarrollada en el lugar de trabajo de uno de los investigadores: la Facultad de Ciencias de la Educación de la Universidad Católica de Maule (Chile), condición que favoreció la posibilidad de llevar a cabo las entrevistas realizadas. Al mismo tiempo, el estudio fue planteado a las au- toridades de la Facultad como una oportunidad para comprender con mayor profundidad los procesos de integración de TIC entre los profesores y, por lo tanto, para mejorar las decisiones que las diferentes unidades académicas que componen la Facultad necesitan tomar para adquirir recursos TIC y definir políticas de formación en el uso de tecnología.

En el caso de este trabajo, debido al interés por comprender y profundizar la visión de los participantes en relación a los temas de la investigación, se optó por la entrevista semiestructurada, planificando previamente un conjunto de preguntas en relación con un guion preestablecido y, al mismo tiempo, permitiendo al entrevistador incorporar preguntas espontáneas.

En relación al tipo de preguntas de la entrevista, se incorporaron reactivos abiertos para entregar un marco de referencia a las respuestas de los entrevistados, estableciendo un mínimo de restricción a las respuestas y a su expresión. Los temas centrales que se abordaron estuvieron relacionados con las expectativas de resultado y de eficacia, y fue-

\begin{tabular}{|c|c|}
\hline Expectativas & Temas \\
\hline De resultados & $\begin{array}{l}\text { Resultados que produce la integración de las TIC en el } \\
\text { proceso de enseñanza-aprendizaje }\end{array}$ \\
\hline \multirow[t]{5}{*}{ De eficacia } & $\begin{array}{l}\text { Hardware y software que el entrevistado se ha propuesto } \\
\text { utilizar }\end{array}$ \\
\hline & $\begin{array}{l}\text { Forma en que se ha propuesto abordar la propiedad } \\
\text { intelectual }\end{array}$ \\
\hline & $\begin{array}{l}\text { Forma en que se ha propuesto integrar los recursos TIC en } \\
\text { el proceso de enseñanza-aprendizaje }\end{array}$ \\
\hline & $\begin{array}{l}\text { Forma en que se ha propuesto integrar recursos TIC para } \\
\text { llevar a cabo sus responsabilidades y tareas profesionales }\end{array}$ \\
\hline & $\begin{array}{l}\text { Forma en que se ha propuesto fomentar el uso de TIC en los } \\
\text { estudiantes para potenciar en ellos responsabilidad, trabajo } \\
\text { en equipo, integración ciudadana e incorporación en el } \\
\text { mundo laboral. }\end{array}$ \\
\hline
\end{tabular}

Tabla 1. Determinación de las preguntas-guía en las entrevistas realizadas. 
ron los expresados en la Tabla 1.

Para determinar el grupo al cual estaba dirigida la entrevista o los destinatarios, se establecieron los siguientes criterios, en función de los objetivos del trabajo y de los propósitos específicos de la investigación:

Académicos pertenecientes a la Facultad de Ciencias de la Educación.

Representantes de varias carreras o unidades académicas.

Manejo del diseño y de la gestión curricular implementado en las carreras de la Facultad de Ciencias de la Educación.

Experiencia y/o vinculación con el sistema escolar.

De los 48 académicos contratados en la Facultad de Educación, diez cumplían con estos requisitos. Finalmente, cinco formaron parte de la muestra, ya que son los que estuvieron dispuestos a participar en el estudio. Estos académicos pertenecían a las siguientes unidades: Escuela de Educación Física, Escuela de Pedagogía en Inglés, Departamento de Fundamentos de la Educación y Decanato. Los académicos que participaron fueron varones entre 48 y 65 años.

\subsection{Procedimiento para el análisis de las entrevistas.}

Tomando en consideración que la metodología cualitativa de la investigación se caracteriza por ser flexible y abierta, y donde la reflexión ocupa un papel fundamental (Bizquerra, 2009), el procedimiento que se ha seguido para decodificar, analizar e interpretar los datos de las entrevistas ha sido el método de la codificación abierta de la teoría fundamentada.

\subsection{Conceptualización y codificación.}

Desde el punto de vista de la teoría fundamentada, se identificaron unidades de registro como parte de un proceso de abstracción y conceptualización, «en el cual los datos se descomponen en incidentes, ideas, acontecimientos y actos discretos a los que luego se les da un nombre que los representa o reemplaza» (Strauss \& Corbin, 2002).

\section{Categorización.}

En el proceso de análisis cualitativo de la información, y una vez que los conceptos codificados comienzan a acumularse, se hace necesario agruparlos en función de un orden abstracto más elevado con el propósito de explicar aquello que está sucediendo. En esto consiste, básicamente, el proceso de categorización, el cual permite al analista reducir la cantidad de unidades con las que trabaja. Para categorizar la información relacionada con las expectativas de resultado y eficacia en torno al uso de las TIC en educación, se optó por seguir un procedimiento análogo al que se utiliza para definir un concepto, entendiendo a éste como una imagen mental por medio de la cual comprendemos las experiencias que emergen de la interacción con nuestro entorno.

Un concepto se origina en la necesidad de generalizar o clasificar las cosas y sus propiedades, agrupándolas a partir de cualidades comunes. De esta manera, somos capaces de designar diversos objetos, hechos y procesos a través de determinadas formas gramaticales y enunciados del habla.

En el caso de la presente investigación, la determinación de categorías para llevar a cabo el análisis cualitativo se estableció a partir de un conjunto de preguntas cuyas respuestas, 


\begin{tabular}{|c|c|c|}
\hline \multirow{4}{*}{$\begin{array}{c}\text { Caracterización de las } \\
\text { expectativas de resultado } \\
\text { y eficacia }\end{array}$} & Pregunta & Categoría \\
\cline { 2 - 3 } & ¿Quién? [integra] & Sujeto \\
\cline { 2 - 3 } & ¿Qué? [integra] & Objeto \\
\cline { 2 - 3 } & ¿Cómo? [integra] & Modo \\
\cline { 2 - 3 } & ¿Para qué? [integra] & Finalidad \\
\hline
\end{tabular}

Tabla 2. Preguntas para definir categorías.

eventualmente, podrían conducir a una definición extensiva del concepto de expectativas de resultado y de eficacia.

Las preguntas que se tomaron como base para definir las categorías están señaladas en la siguiente Tabla 2.

Una vez establecidas las categorías de análisis para la parte cualitativa del estudio, las sub-categorías fueron determinadas de manera inductiva, a partir del proceso de codificación y clasificación de las unidades de registro, identificadas en el corpus de texto que se obtuvo de la transcripción de las entrevistas.

El esquema de categorías expresado en la Tabla 3 es el que finalmente fue utilizado para llevar a cabo el análisis de la información cualitativa recogida a través de las entrevistas semi-estructuradas, que se aplicaron de manera individual a los informantes de la investigación.

\subsection{Uso del programa computacional ATLAS.ti para el análisis cualitativo.}

ATLAS.ti es un programa especialmente desarrollado para la elaboración de teoría entrañada (Navarro \& Díaz, 1994). En primer lugar, permite reunir documentos, a los cuales se les denomina unidades hermenéuticas. Dentro de estas unidades hermenéuticas, ayuda a marcar y segmentar textos, facilitando la comparación entre segmentos textuales y su posterior codificación (unidades de registro adscritas a unidades de contexto). ATLAS.ti facilita la elaboración de un análisis cualitativo a partir de su particular sistema de codificación, que se basa en categorías semánticas y familias de categorías. Al mismo tiempo permite visualizar de manera gráfica (networks) las conexiones entre fragmentos textuales, comentarios, códigos y conceptos.

\section{Resultados.}

\subsection{Integración TIC en educación desde la actividad del sujeto.}

Al hablar de la actividad del sujeto en relación al uso de las TIC distinguimos, básicamente, la actividad que lleva a cabo el(la) estudiante y la que lleva a cabo el(la) profesor(a). A pesar de que la visión de los entrevistados no es homogénea en este punto, prevalece una mirada que considera un rol pasivo del sujeto en cuanto al uso de las TIC. El centro está puesto en los contenidos y las TIC son elementos que permiten que el sujeto los incorpore o los transmita de mejor manera, dependiendo si se trata del profesor o del estudiante. El uso de las TIC es un imperativo que va más allá de la voluntad o la intención de las personas que las utilizan. Los métodos frontales de enseñanza, basados en la trans- 


\begin{tabular}{|c|c|c|c|}
\hline CATEGORÍA & SUBCATEGORÍA & CÓDIGO & DESCRIPCIÓN \\
\hline \multirow[b]{2}{*}{$\begin{array}{l}\text { SU-Expectativas } \\
\text { hacia el sujeto }\end{array}$} & $\begin{array}{l}\text { 04-Integración TIC } \\
\text { en las tareas del } \\
\text { alumno }\end{array}$ & $\begin{array}{l}\text { SU04-Efecto aprendizaje } \\
\text { SU04-Grabaciones y registros } \\
\text { SU04-Participación en foros } \\
\text { SU04-Realización vídeos } \\
\text { SU04-Trabajo en equipo mediante TIC }\end{array}$ & \multirow{2}{*}{$\begin{array}{l}\text { Cómo es percibido el papel que tien } \\
\text { el uso de las TIC, desde el punto de } \\
\text { vista de la actividad del sujeto, } \\
\text { identificando los dos sujetos } \\
\text { fundamentales en el proceso de } \\
\text { enseñanza-aprendizaje: profesor y } \\
\text { alumno. }\end{array}$} \\
\hline & $\begin{array}{l}\text { 05-Integración TIC } \\
\text { en las tareas } \\
\text { docentes }\end{array}$ & $\begin{array}{l}\text { SU05-Apoyo trabajo directivo } \\
\text { SU05-Deber del docente } \\
\text { SU05-Deber integración profesor } \\
\text { SU05-Redes sociales } \\
\text { SU05-Tareas transversales } \\
\text { SU05-Uso de guías } \\
\text { SU05-Uso pedagógico TIC } \\
\text { SU05-Uso plataformas virtuales } \\
\text { SU05-Vinculación estudiantes }\end{array}$ & \\
\hline $\begin{array}{l}\text { OB- } \\
\text { Expectativas } \\
\text { hacia el objeto }\end{array}$ & $\begin{array}{l}\text { 07-Recursos } \\
\text { preferidos }\end{array}$ & $\begin{array}{l}\text { OB07-Notebook } \\
\text { OB07-Ofimática } \\
\text { OB07-Participación alumnos mediante } \\
\text { TIC } \\
\text { OB07-Presentaciones } \\
\text { OB07-Recursos disponibles } \\
\text { OB07-Redes sociales } \\
\text { OB07-Uso de Internet } \\
\text { OB07-Uso de presentaciones } \\
\text { OB07-Vídeo conferencia }\end{array}$ & $\begin{array}{l}\text { Cuáles son los recursos que se } \\
\text { mencionan cuando se habla de la } \\
\text { incorporación de las TIC en la } \\
\text { educación. Se refiere a las } \\
\text { herramientas de hardware y de } \\
\text { software que se utilizan. }\end{array}$ \\
\hline \multirow{3}{*}{$\begin{array}{l}\text { MO- } \\
\text { ExpectativasMo } \\
\text { do de uso }\end{array}$} & $\begin{array}{l}\text { 03-Integración TIC } \\
\text { en aprendizajes }\end{array}$ & $\begin{array}{l}\text { MO03-Apoyo tareas } \\
\text { MO03-Aprendizaje por } \\
\text { descubrimiento } \\
\text { MO03-Creación material didáctico } \\
\text { MO03-Habilidades mediante TIC } \\
\text { MO03-Transversalidad }\end{array}$ & \multirow{3}{*}{$\begin{array}{l}\text { La manera en que las TIC son } \\
\text { integradas en el proceso de } \\
\text { enseñanza-aprendizaje. Cómo son } \\
\text { utilizadas para desarrollar y potencia } \\
\text { el aprendizaje del estudiante y de qu } \\
\text { manera son atendidas las condicione } \\
\text { legales de propiedad intelectual, } \\
\text { asociadas a su uso. }\end{array}$} \\
\hline & $\begin{array}{l}\text { 09-Propiedad } \\
\text { intelectual de } \\
\text { contenidos }\end{array}$ & $\begin{array}{l}\text { MO09-importancia propiedad } \\
\text { intelectual } \\
\text { MO09-Limitaciones propiedad } \\
\text { intelectual } \\
\text { MO09-Mundo globalizado } \\
\text { MO09-Trabajo intelectual alumnos } \\
\text { MO09-Uso de recursos disponibles }\end{array}$ & \\
\hline & $\begin{array}{l}\text { O8-Propiedad } \\
\text { intelectual de } \\
\text { software }\end{array}$ & $\begin{array}{l}\text { MO08-Software libre } \\
\text { MO08-Software propietario } \\
\text { MO08-uso software originales }\end{array}$ & \\
\hline \multirow{3}{*}{$\begin{array}{l}\text { FI- } \\
\text { ExpectativasFin } \\
\text { alidad }\end{array}$} & $\begin{array}{l}\text { O6-Necesidad en el } \\
\text { uso de las TIC }\end{array}$ & $\begin{array}{l}\text { FI06-Analfabetismo tecnológico } \\
\text { FI06-Integración no opcional } \\
\text { FI06-Necesidad de uso }\end{array}$ & \multirow{3}{*}{$\begin{array}{l}\text { Para qué son utilizadas las TIC en el } \\
\text { ámbito educativo. El aporte que esta } \\
\text { herramientas constituyen para la } \\
\text { educación, para qué y cómo se } \\
\text { necesitan y la funcionalidad que } \\
\text { ofertan. }\end{array}$} \\
\hline & $\begin{array}{l}\text { 02-Impacto de las } \\
\text { TIC }\end{array}$ & $\begin{array}{l}\text { FI02-Aprendizaje novedoso } \\
\text { FI02-Condición impacto } \\
\text { FI02-Duda del impacto }\end{array}$ & \\
\hline & $\begin{array}{l}\text { O1-Funcionalidad de } \\
\text { las TIC }\end{array}$ & $\begin{array}{l}\text { FI01-Actualización a través de las TIC } \\
\text { FI01-Grabaciones y registros } \\
\text { FI01-Potencialidad TIC } \\
\text { FI01-Tipo herramientas } \\
\text { FI01-Utilidad relativa TIC }\end{array}$ & \\
\hline
\end{tabular}

Tabla 3. Análisis de la información cualitativa. 
misión de información a través de los libros o de la pizarra tradicional, siguen siendo efectivos para el aprendizaje, pero no son novedosos ni atrayentes. Por tanto, el valor de las tecnologías está en que permiten generar estímulos más potentes, más impactantes. En absoluto se pone en duda el paradigma conductista estímulo-respuesta.

Otro resultado importante a considerar es que se considera que la enseñanza tiene la función de preparar a los estudiantes para lo que hay, para lo que existe, no para formar criterios que permitan discernir en relación a lo que es más apropiado de acuerdo a un determinado propósito o a un determinado objetivo.

\subsection{Las TIC como objetos-herramientas en el ámbito educativo.}

En términos generales, las herramientas TIC mencionadas por los entrevistados se limitan a programas básicos de ofimática, algunas aplicaciones de comunicación en Internet y equipos asociados a la captura y reproducción de sonido y vídeo. Incluso hay respuestas que reflejan desconocimiento del concepto de TIC, identificando como tales objetos que desde ninguna perspectiva entran en esta categoría.

El programa PowerPoint, como herramienta para la exposición de contenidos, prevalece indiscutiblemente sobre otro tipo de software, aunque también se menciona Word, Excel y aplicaciones de comunicación en Internet como el correo electrónico y las redes sociales. De todos modos, apenas se menciona el uso específico que se lleva a cabo a través de este tipo de recursos.

Es posible inferir que esta poca diversidad en la utilización de recursos TIC se corresponde con la forma plana y tendente a la trans- misión frontal de contenidos en que estas herramientas son incorporadas en el proceso de enseñanza-aprendizaje.

\subsection{Modo en que las TIC son integradas en educación.}

De acuerdo con los códigos y las categorías de análisis utilizados, los resultados apuntan a que el modo en que las TIC son integradas en educación se vincula con el uso que hacen los profesores de ellas en el proceso de aprendizaje de los estudiantes y la forma en que abordan los aspectos vinculados a la propiedad intelectual, tanto de herramientas (software) como de contenidos digitales.

Los profesores entrevistados declaran utilizar las TIC para fomentar el aprendizaje en sus estudiantes. Un académico de la carrera de Inglés y otro de la carrera de Educación Física describieron actividades específicas, en las cuales las TIC cumplían un papel importante dentro de la estrategia de enseñanza que decían implementar. Sin embargo, en términos generales, los profesores mencionaron un uso genérico, en el cual no se definía una aplicación concreta de estos recursos, ya fuera para planificar, implementar una clase o evaluar a los estudiantes.

Respecto a las cuestiones relacionadas con la propiedad intelectual, es posible apreciar un gran desconocimiento de la forma en cómo opera la propiedad intelectual hacia los recursos de software y hacia los contenidos digitales disponibles en Internet. Se tiende, por ejemplo, a mostrar la propiedad intelectual como un derecho que defiende la producción que llevan a cabo los mismos estudiantes mediante sus trabajos, pero se omiten las restricciones que esta forma de propiedad impone hacia muchas de las herramientas de software que se utilizan para cons- 
truir los productos desarrollados por estudiantes y profesores. En este sentido, se le da importancia a la protección de los contenidos que se generan o a los cuales se accede a través de Internet, pero las condiciones privativas de licencia de las aplicaciones que más se utilizan para trabajar son pasadas por alto. Es así, por ejemplo, cómo un número importante de profesores y alumnos tienen instalados en sus ordenadores personales software que vulnera dichas condiciones de licencia porque no ha sido comprado. El caso más notorio y abundante lo constituyen las herramientas de Microsoft Office conseguidas y copiadas entre amigos.

Es difícil considerar una adecuada integración de las TIC en el proceso educativo, si no se atiende un aspecto tan fundamental como las definiciones legales de propiedad de las herramientas y de la información que se está utilizando para aprender, para enseñar o para generar nueva información y nuevo conocimiento con fines intelectuales o académicos.

\subsection{Finalidad en el uso e integración de las TIC.}

La finalidad en el uso de la integración de las TIC será abordada desde tres puntos de vista: la necesidad, el impacto y la funcionalidad de las TIC en el ámbito educativo.

La necesidad de integrar las TIC en el proceso de enseñanza-aprendizaje, según los resultados obtenidos, tiene un carácter de algo imperativo, más allá de las intenciones, voluntades, deseos o aspiraciones de los sujetos involucrados. Las TIC son objetos que permiten a las personas no quedar obsoletas y se considera que aunque los métodos tradicionales sigan siendo «eficaces», las nuevas tecnologías tienen un impacto sobre la motivación y el interés de los estudiantes. Nótese que de manera implícita se da por hecho que la motivación de los estudiantes es extrínseca, dependiendo de estímulos apropiados que, en este caso, son entregados por las tecnologías, y no a partir de objetivos, metas o propósitos que provienen del sujeto y que pueden ser compartidos con los demás en espacios comunes de relación e interacción. Si el sujeto no recibe estos estímulos puede perder el «encanto». Subyace una mirada que considera una acción educativa orientada hacia individuos, separados unos de otros, que reaccionan y que esencialmente son pasivos, requiriendo ser «encantados» para modificar sus conductas (aprender).

Aparentemente el uso de las TIC impacta positivamente en el aprendizaje, aunque uno de los entrevistados sugiere que no hay ningún estudio que respalde de manera definitivamente este supuesto.Otro de los profesores entrevistados considera que las TIC funcionan como un lenguaje de carácter universal: quien no sabe utilizarlas es un analfabeto, sobretodo en el tipo de sociedad en el que vivimos.

\subsection{Representación gráfica del contenido de las entrevistas.}

A continuación, en el siguiente esquema (ilustración $n^{\circ} 1$ ) se presenta la estructura de codificación y categorías utilizadas para organizar el contenido de las entrevistas: 


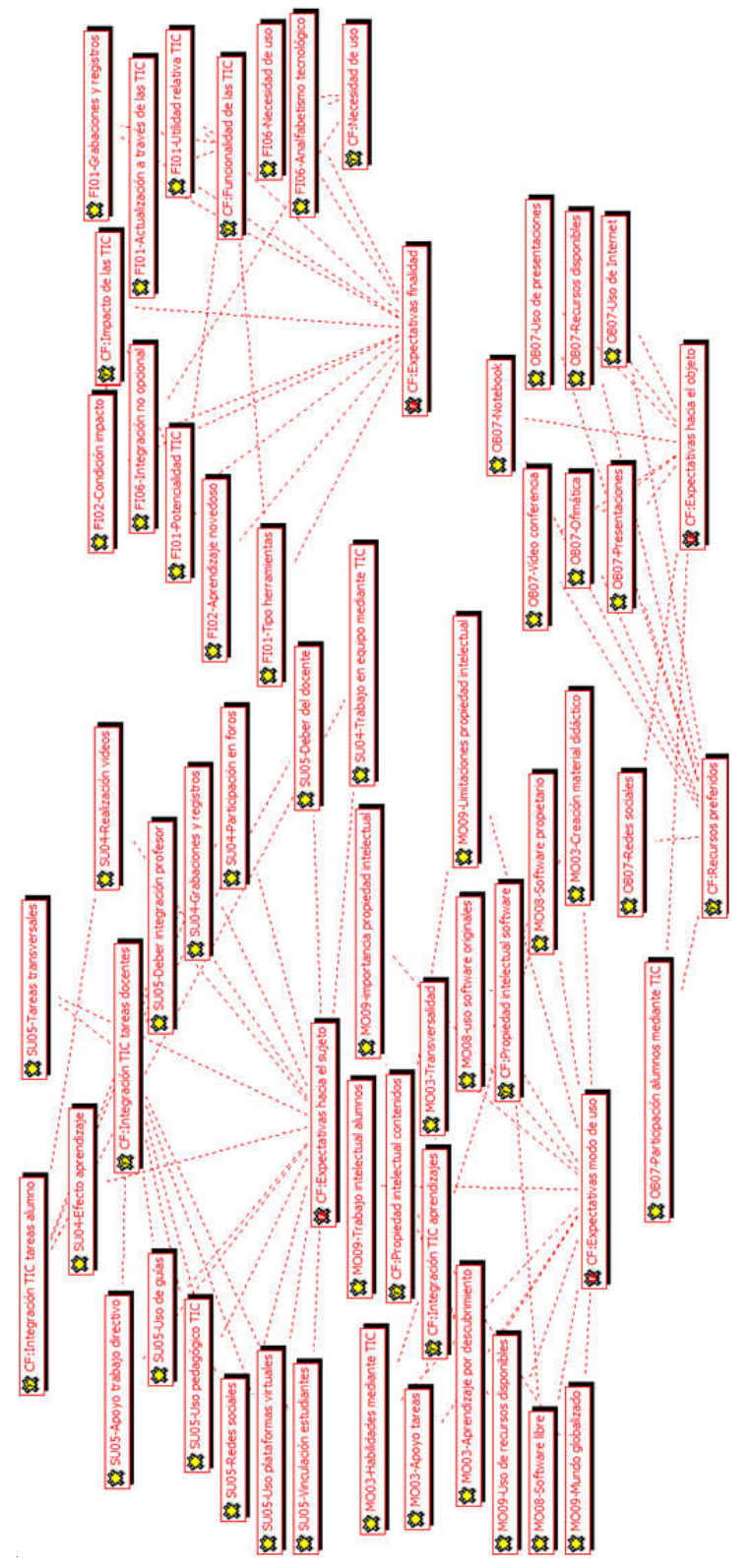

Ilustración 1: Codificación y categorías utilizadas $-60-$

Píxel-Bit. Revista de Medios y Educación. No 46. Enero 2015. ISSN: 1133-8482. e-ISSN: 2171-7966. doi: http://dx.doi.org/10.12795/pixelbit.2015.i46.04 


\section{Conclusiones.}

Los resultados obtenidos en este trabajo nos permiten constatar que los académicos de todas las carreras de Pedagogía en la UCM poseen altas expectativas acerca de los efectos que se producen al utilizar las TIC, ya sea para facilitar la manera en que se enseña o en que se aprende, para aumentar el interés y la motivación, para adaptarse a las demandas de formación que plantea la sociedad actual o para conseguir el reconocimiento externo en relación a los roles que se deben desempeñar en el proceso educativo, resultados que concuerdan con los estudios realizados por (Ifeoma, 2014; Kapoor \& Sharma, 2013; Koc $\&$ Bakir, 2010).

Por otro lado, cabe decir que el valor de las tecnologías es atribuido a que permiten generar estímulos más potentes y más impactantes, tal y como apuntan Oostveen, Murirhead y Goodman (2011) y Zelick (2013). Predomina un paradigma conductista estímulo-respuesta, que considera la motivación como algo extrínseco a la intencionalidad del sujeto. Para esta mirada, lo más importante en educación es que se entreguen los estímulos apropiados, no que se construyan objetivos, metas y propósitos comunes que involucren a los sujetos. En este sentido, existe una gran confianza en los objetos, en el equipamiento y en las herramientas, más que en los sujetos que las utilizan. Esta idea es coherente con lo que plantean Valverde, Garrido y Sosa (2010) acerca de la percepción del profesorado innovador en torno a los impactos de las TIC en el proceso educativo, específicamente en el papel que tienen las TIC para promover la creatividad, la autonomía del estudiante, la adquisición de aprendizajes significativos, la superación de dificultades en el aprendizaje, la conexión de ideas y la ampliación del saber. Según estos autores, las políticas educativas para fomentar la incorporación de las tecnologías de la información y la comunicación en el aula se han orientado a mejorar el equipamiento y la infraestructura de los centros educativos, pero no han conseguido generar un impacto educativo de fondo en el proceso de enseñanzaaprendizaje. Asimismo, las investigaciones de (Benavides \& Francesc, 2007; Olvera-Lobo \& Benítez-de-Vendrell, 2008; Tejedor, GarcíaValcárcel \& Prada, 2009) vienen a corroborar los resultados del presente estudio, en el sentido de que existe una marcada tendencia a dar prioridad a los objetos sobre los sujetos, en el uso e incorporación de tecnologías en educación, aunque la evidencia nos muestra que una disposición adecuada en relación al uso de las TIC incide en la eficacia que pueden tener estos recursos en el proceso formativo, sin importar demasiado lo sofisticada que pueda ser esta tecnología (Olvera-Lobo $\&$ Benítez-de-Vendrell, 2008).

La adquisición de estas herramientas y, especialmente, de aquellas que en un determinado momento se presentan como «lo más avanzado», constituye un imperativo, un camino obligado, para que los países y las instituciones no queden obsoletas (Benavides \& Francesc, 2007; Hayes \& Orellana, 2011), pero no sólo los países y las instituciones, sino también los pueblos y las personas (Almekhlafi \& Almeqdadi, 2010). De esta manera cobra fuerza el concepto de obsolescencia programada y percibida, base ideológica, a partir de los años 50, de la sociedad capitalista de consumo (Castells, 2001), que bien se sintetiza en la frase incorporada por Anny Leonard en su documental Story of Stuff, citando al analista de mercado Víctor Lebow:«nuestra economía, enormemente productiva, exige que hagamos del 
consumo nuestro estilo de vida, que convirtamos el comprar y utilizar bienes, en auténticos rituales, que busquemos nuestra satisfacción espiritual, la satisfacción del ego, en el consumir... necesitamos que se consuman cosas, se quemen, se sustituyan, y se tiren, todo ello a un ritmo cada vez más rápido» (Leonard, 2007).

En este sentido, las TIC están dentro de los bienes de consumo que, probablemente, se encuentran más determinados por el fenómeno de la obsolescencia. Los resultados en este estudio han demostrado que si las expectativas de resultado no son acompañadas por un proceso de reflexión que permita entender la incorporación de las TIC en la sociedad desde una perspectiva integral, tal y como también apuntan Aguaded-Gómez y Pérez-Rodríguez (2012), en lugar de convertirse en un beneficio, habrán de favorecer, enormemente, este deseo de comprar, tirar y reemplazar, característicos de una sociedad de consumo, con todas las consecuencias humanas y medioambientales que hoy en día nos afectan.

\section{Fuentes de financiación.}

Beca estancia corta de Post-doctorado: Fundación Carolina. Además, el presente trabajo se enmarca en el seno del Grupo de Investigación «EDUTIC-ADEI» (Ref.: Vigrob039) y del Programa de Redes de investigación en docencia universitaria del ICE, todos ellos de la Universidad de Alicante (UA); del proyecto Instituto Superior de Investigación Cooperativa IVITRA (Ref.: ISIC/2012/022; http://www.ivitra.ua.es) y del Proyecto DIGICOTRACAM («Programa Prometeo de la Generalitat Valenciana para Grupos de Investigación en I+D de Excelencia», Ref.: Prometeo-2009-042, cofinanciado por el
FEDER de la UE) y MICINN FFI2009-13065 y FFI2010-09064.

\section{Bibliografía.}

Aguaded-Gómez, I.\& Pérez-Rodríguez, M. (2012). Strategiesfor media literacy: Audiovisual skills and the citizenship in Andalusia. Journal of New Approaches in Educational Research, 1(1), 22-26. doi:10.7821/naer.1.1.22-26

Almekhlafi, A. G. \& Almeqdadi, F. A. (2010).Teachers' Perceptions of Technology Integration in the United Arab EmiratesSchool Classrooms. Educational Technology \& Society, 13 (1), 165-175.

Benavides, F. \& Francesc, P. (2007).Políticas Educativas sobre Nuevas Tecnologías en los Países Iberoamericanos. Revista Iberoamericana de Educación, 45, 19-69.

Bizquerra, R. (2009). Metodología de la Investigación Educativa. Madrid: Editorial la Muralla.

Cabero Almenara, J. \& Marín Díaz, V. (2012). ICT training of university teachers in a personal learning environment. Project DIPRO 2.0. Journal of New Approaches in Educational Research, 1 (1), 2-6. doi:10.7821/ naer.1.1.2-6

Castells, M. (2001). La Era de la Información: La Sociedad Red (Vol. I). Madrid: Alianza Editorial.

Chiner, E. \& Cardona, M. C. (2012). Teachers' use of inclusive practices in Spain. The International Journal of Learner Diversity and Identities, 19 (1), 29-45.

Chiner, E. \& Cardona, M. C. (2013). Inclusive education in Spain: How do skills, resources, and supports affect regular education teachers' perceptions of inclusion?. The International Journal of Inclusive Education, 17 (5), 526-541.doi: 


\subsection{0/13603116.2012.689864}

Hayes Capo, B. \& Orellana, A. (2011).Web 2.0 technologies for classroom instruction. High School Teachers' Perceptions and Adoption Factors. The Quarterly Review of Distance Education, 12 (4), 235-253.

Ifeoma Asiyai, R. (2014). Assessment of Informationand Communication Technology Integration in Teaching and Learning in Institutions of Higher Learning. International Education Studies, 7 (2), 25-36.doi:10.5539/ ies.v7n2p25

Kapoor, T. \& Sharma, M. (2013). A factor analysis of attitude of education fraternity towards the role of technology ineffective learning: an empirical study in higher education institution. Signum Temporis. Journal of Pedagogy and Psychology, 6 (1), 4-16.doi: 10.2478/sigtem-2013-0056

Koc, M. \& Bakir, N. (2010). A needs assessment survey to investigate pre-service teachers'knowledge, experiences and perceptions about preparation tousing educational technologies.TOJET: The Turkish Online Journal of Educational Technology, 9 (1), 13-22.

Leonard, A. (2011). Story of Stuff. Recuperado dehttp://storyofstuff.org/.

López-Torrijo, M. \& Mengual-Andrés, S. (2014). Students with severe, permanent disabilities and their educational inclusion in Spain. International Education Studies, 7 (2), 91-105. doi: 10.5539/ies.v7n2p91 Loyd, B. H.\&Gressard, C. (1984). Reliability and factoral validity of computer attitude scale. Educational and Psychological Measurement, 44 (2), 501-505. doi: 10.1177/ 0013164484442033

Marín Díaz, V. \& Martín-Párraga, J. (2014). Can videogames be used to develop the infant stage educational curriculum?. Journal of New Approaches in Educational Research,
3 (1), 20-25. doi:10.7821/naer.3.1.20-25

Navarro, P. \& Díaz, C. (1994). La confrontación de modelos y niveles epistemológicos en la génesis e historia de la investigación social. En J.M. Delgado y J. Gutiérrez (Eds.) Métodos y técnicas cualitativas de investigación en las ciencias sociales. (pp. 213). Madrid: Editorial Síntesis.

Olvera-Lobo, M. \& Benítez-de-Vendrell, B. (2008). Aproximación a las actitudes y percepciones de los usuarios ante las tecnologías de la información. El profesional de la información, 17 (2), 199-204. doi: 10.3145/ epi.2008.mar.10

Oostveen, R., Muirhead, W. \& Goodman, W. M. (2011). Tablet PCs and reconceptualizing learning with technology: a case study in higher education. Interactive Technology and Smart Education, 8 (2), 7893. doi 10.1108/17415651111141803.

Pieron, Henri. (1993). Vocabulario Akal de Psicología. Madrid: Ediciones Akal.

Saeed, N., Yang, Y. \& Sinnappan, S. (2009). Emerging Web Technologies in Higher Education: A Case of Incorporating Blogs, Podcasts and Social Bookmarks in a Web Programming Course based on Students' Learning Styles and Technology Preferences. Educational Technology \& Society, 12 (4), 98-109.

Strauss, A. \& Corbin, J. (2002). Bases de la Investigación Cualitativa.Madellín: Editorial Universidad de Antioquía.

Tejedor, F.J., García-Valcárcel, A. \& Prada, S. (2009). Medida de Actitudes del Profesorado Universitario hacia la Integración de las TIC. Comunicar, Revista Científica de Educomunicación, 33, 115-124. doi:10.3916/ c33-2009-03-002

Valverde, J., Garrido M. \& Sosa M. (2010). Políticas educativas para la integración de las TIC en Extremadura y sus efectos sobre la 
innovación didáctica y el proceso enseñanza-aprendizaje: la percepción del profesorado. Revista de Educación, 352, 99-124.

Yau, H. K. \& Cheng, A. (2012).Students' age difference of confidence in using technology for learning in higher education. TOJET: The Turkish Online Journal of Educational Technology, 11 (3), 308-311.

Zelick, S. A. (2013). The Perception of Web 2.0 Technologies on Teaching and Learning in Higher Education: A Case Study. Creative Education, 4 (7B), 53-93. doi: 10.4236/ ce.2013.47A2010.

Fecha de recepción: 31-01-2014 Fecha de evaluación: 18-03-2014

Fecha de aceptación: 28-04-2014 